\title{
NONINVASIVE VENTILATION IN PATIENTS WITH SLEEP APNEA SYNDROME COMPLICATED WITH GERD.
}

1. MBBS, FCPS (Pulmonology) Assistant Professor

Department of Pulmonology Faisalabad Medical University

2. MBBS, FCPS (Anesthesia)

Assistant Professor Department of Anesthesia

Faisalabad Medical University, Faisalabad.

3. MBBS, FCPS (Anesthesia) Assistant Professor Department of Anesthesia Independent Medical College Faisalabad.

Correspondence Address: Dr. Mohsin Riaz Askri

Department of Anesthesia Faisalabad Medical University, Faisalabad.

mohsinriaz46@yahoo.com

Article received on: 07/05/2019

Accepted for publication: $15 / 08 / 2019$

\begin{abstract}
Umar Usman', Mohsin Riaz Askri², Shumyala Maqbool ${ }^{3}$
ABSTRACT... Objectives: The access the clinical effects of non-invasive ventilation in patients with obstructive sleep apnea (OSA) accompanied with GERD. Study Design: Randomized Controlled Trial. Setting: Department of Pulmonology, DHQ Teaching Hospital Faisalabad. Periods: January 2018 to December 2018. Material \& Methods: As Stated in sleep study results, 100 patients with OSA were divided into three different groups according to the disease severity that is mild, moderate, and severe groups. Gastroscopy was performed in all the patients and underwent GERD assessment. Twenty-three patients with OSA accompanied with GERD were segregated into an experimental group and control group, who were given omeprazole (40 mg/time per day) combined with non-invasive ventilation therapy and omeprazole (40 mg/ time per day) alone, respectively, for eight weeks. After that, they Underwent gastroscopy and GERD assessment. Results: Among all the 100 patients with OSA, 48 were in the mild group, 33 in the moderate group, and 19 in the severe group. Their GERD scores were $8.26 \pm 1.11$, $9.87 \pm 1.79$, and $12.34 \pm 2.02$, respectively, with a statistical difference $(P=0.004)$. Out of 23 patients with OSA associated with GERD were divided into The Experimental Group, which included 12 patients, and Control Group, which included. 11 patients. The gastroscopy efficacy rates for inflammation management were $86.53 \%$ and $53.09 \%$, respectively, with a statistical difference $(P=0.011)$. The $\triangle$ GERD scores for the two groups also differed significantly $(5.11 \pm$ 1.54 vs $2.35 \pm 0.72, P=0.034)$. Conclusion: Patients having severe OSA were associated with a high incidence rate of GERD than those with moderate or mild OSA. Those who were treated with non-invasive ventilation combined with PPI shown much better results than PPI alone in respect of clinical effects in patients with OSA associated with GERD.
\end{abstract}

Key words: $\quad$ Gastroesophageal Reflux Disease, Noninvasive Ventilation, Obstructive Sleep Apnea.

Article Citation: Usman U, Askri MR, Maqbool S. Noninvasive Ventilation in patients with Sleep Apnea syndrome complicated with GERD. Professional Med J 2020; 27(1):120-124. DOI: 10.29309/TPMJ/2020.27.1.3681

\section{INTODUCTION}

Obstructive sleep apnea (OSA) is a severe sleepdisordered breathing disorder that is clinically common, with hypoventilation and apnea during sleep, with or without snoring. As the main features, the main pathological manifestations are intermittent hypoxemia and / or hypercapnia and sleep structural disorders, causing damage to a variety of organs or systems, followed by a variety of complications, such as elevated blood pressure, cardiovascular and cerebrovascular Disease, pulmonary hypertension and pulmonary heart disease, insulin resistance and type 2 diabetes, gastro esophageal reflux disease (GERD), sexual dysfunction, kidney damage, cognitive impairment, etc. ${ }^{1-3}$ GERD $^{4}$ It means that the contents of the stomach and duodenum are reversed into the esophagus, and then symptoms such as acid reflux, chest pain, heartburn, and bloating occur, and some patients may have symptoms other than the esophagus. According to the results of electronic gastroscopy, GERD can be divided into Reflux esophagitis (RE) and non-erosive reflux disease (NERD), RE refers to esophageal mucosal congestion seen under gastroscopy, edema, with esophageal mucosal erosion; NERD refers to patients with normal GERD disease under gastroscopy esophageal mucosa. It has been reported that obstructive sleep apnea hypopnea syndrome is associated with GERD ${ }^{5-7}$ due to airway obstruction in patients with obstructive sleep apnea, pleural negative 
pressure and negative esophageal pressure will increase significantly during inhalation, which in turn causes gastric contents to flow back into the esophageal injury esophageal mucosa. In addition, OSA The nighttime micro-awakening state and increased swallowing movements lead to transient relaxation of the upper esophageal sphincter, which is also an important mechanism leading to reflux. The incidence of OSA combined with GERD is gradually increasing, which has become a common clinical problem. There is no standard treatment plan. This article discusses the therapeutic effect of non-invasive ventilation in patients with OSA combined with GERD, in order to provide reference for the clinical diagnosis and treatment of such diseases.

\section{MATERIAL}

100 patients with obstructive sleep apnea who were admitted to the Department of pulmonology DHQ Hospital Faisalabad, 2017-12/2018-02, were all conducted with sleep study, female 41 For example, 59 males, aged 28-63 years, mean age 42.4 years \pm 7.6 years, the diagnostic criteria are consistent with the "Guidelines for the diagnosis and treatment of obstructive sleep apnea (basic version)"1 All patients have signed an informed situation consent.

\section{METHODS}

100 patients with OSA were divided into mild, moderate, and severe according to the results of sleep study. They were also examined by electronic gastroscopy and GERD scale (Table-I). According to the results of gastroscopy, they were divided into RE and NERD. Divided into experimental group and control group, based on health education for all patients (guide diet / reasonable rest / smoking cessation, etc.), the experimental group was given omeprazole (40 mg / time- d) Combined with non-invasive ventilation, the control group was given omeprazole $(40 \mathrm{mg} /$ day). The patients in both groups were treated with gastroscopy and GERD scale after 8 weeks of treatment.

\begin{tabular}{|l|c|c|c|c|}
\hline & \multicolumn{5}{|c|}{ Recalling the Past (d) } \\
\hline Heartburn & 0 d & $\mathbf{1}$ d & $\mathbf{2 - 3}$ d & $\mathbf{4 - 7}$ d \\
\hline Acid reflux & 0 & 1 & 2 & 3 \\
\hline Upper abdominal pain & 0 & 1 & 2 & 3 \\
\hline Nausea & 3 & 2 & 1 & 0 \\
\hline sleep disorder & 3 & 2 & 1 & 0 \\
\hline Using OTC drugs & 0 & 1 & 2 & 3 \\
\hline \multicolumn{5}{|r|}{ Table-I. GERDQ scale. } \\
\hline
\end{tabular}

The GERD scale is used to assess the frequency of attacks in the past 1 week, such as heartburn, reflux, upper abdominal pain, nausea, sleep disorders, and whether or not to use over-thecounter drugs. The frequency of attacks is $0 \mathrm{~d}$, $1 \mathrm{~d}, 2$-3 d, 4-7 d are recorded as 0, 1, 2, and 3 , respectively. The total score is 18 points. The GERDQ score of the test is the sum of the above episode frequency integrals. 8 is divided into diagnostic thresholds. OTC: Over-the-counter drugs.

According to the degree of endoscopic esophageal mucosal injury, GERD is divided into four grades A, B, C, and D. Grade A esophagitis refers to the damage of the esophageal mucosa is confined to mucosal folds, unfused; and the length of erosion is $<5 \mathrm{~mm}$, Grade $\mathrm{E}$ eruption length of esophagitis is $>5 \mathrm{~mm}$; C-level esophagitis has a fusion of esophageal injury, but does not exceed $75 \%$ of esophageal circumference; Grade D esophagitis refers to mucosal damage around the esophagus. ${ }^{8}$ Efficacy judgment: Cure: Esophageal mucosal inflammation disappeared; improved: Esophageal mucosal inflammation was relieved above grade I; Ineffective: Endoscopic inflammation showed no change before and after treatment. Effectiveness = (healing + improvement) / total number of cases. GERD scale score before and after treatment was changed by $\triangle$ GERD Table scores indicate, $\triangle G E R D$ scale score $=$ pre-treatment score - posttreatment score.

Experimental instruments: gastroscopy, Sleep study (Philips Alice LE, non-invasive ventilator (RESMED dual-level non-invasive ventilator VPAP III ST-A). 


\section{Statistical Analysis}

The measurement data were expressed as mean \pm SD. One-way analysis of variance was used for comparison between groups. The q- test was used for comparison between groups. SPSS 17.1 statistical software was used to assist the statistical data. $P$ value $<0.05$ was used for the difference. Significance.

\section{RESULTS}

2.1 GERDQ scale score and RE incidence rate in patients with different degrees of sleep apnea syndrome of the 100 patients with sleep apnea syndrome, 48 were in the mild OSAHS group, 33 in the moderate OSA group, and 19 in the severe OSA group. The GERD scale scores were 8.26 $\pm 1.11,9.87 \pm 1.79,12.34$, respectively. \pm 2.02 , the three groups were statistically different, the $P$ value was 0.27 , and the incidence of $R E$ was $8.33 \%, 21.21 \%$, and $63.16 \%$, respectively. There was a statistical difference between the three groups, P value was 0.004 (Table-II).

\begin{tabular}{|l|c|c|c|c|}
\hline & $\begin{array}{c}\text { Mild } \\
\text { OSA }\end{array}$ & $\begin{array}{c}\text { Moderate } \\
\text { OSA }\end{array}$ & $\begin{array}{c}\text { Severe } \\
\text { OSA }\end{array}$ & P-Value \\
\hline $\begin{array}{l}\text { GERDR } \\
\text { scale } \\
\text { score }\end{array}$ & $\begin{array}{c}8.26 \pm \\
1.11\end{array}$ & $\begin{array}{c}9.87 \pm \\
1.79\end{array}$ & $\begin{array}{c}12.34 \pm \\
2.02\end{array}$ & 0.027 \\
\hline $\begin{array}{l}\text { RE } \\
\text { incidence } \\
\text { rate (\%) }\end{array}$ & 8.33 & 21.21 & 63.16 & 0.004 \\
\hline
\end{tabular}

Table-II. GERDQ scale scores and incidence of reflux esophagitis in patients with different obstructive sleep apnea syndrome.

\section{OSAHS}

Obstructive sleep apnea hypopnea syndrome; RE: reflux esophagitis. 2.2 Endoscopic inflammatory manifestations and changes in GERDQ scale scores in patients with obstructive sleep apnea syndrome complicated with RE Twenty-three patients with OSA combined with GERD, 12 in the experimental group and 11 in the control group, the endoscopic inflammation rates were $86.53 \%$ and $53.09 \%$, respectively. The difference between the two groups was statistically significant, $P$ value was 0.011 . The $\triangle$ GERD amount the table scores were $5.11 \pm 1.54$ and $2.35 \pm 0.72$, respectively. Difference between these two groups were statistically significant, with a $P$ value of 0.034
(Table-III).

\begin{tabular}{|l|c|c|c|}
\hline & Test Group & $\begin{array}{c}\text { Control } \\
\text { Group }\end{array}$ & P-Value \\
\hline $\begin{array}{l}\text { Endoscopic } \\
\text { inflammation is } \\
\text { effective (\%) }\end{array}$ & 86.52 & 53.09 & 0.011 \\
\hline $\begin{array}{l}\Delta \text { GERDQ scale } \\
\text { score }\end{array}$ & $5.11 \pm 1.54$ & $2.35 \pm 0.72$ & 0.034 \\
\hline $\begin{array}{l}\text { Table-III. Endoscopic inflammation efficiency and } \\
\text { GERDQ scale scores in patients with two obstructive } \\
\text { sleep apnea with reflux esophagitis. }\end{array}$ \\
\hline
\end{tabular}

$\triangle$ GERDQ scale score $=$ pre-treatment score post-treatment score.

\section{DISCUSSION}

In recent years, the incidence of obstructive sleep apnea and GERD has gradually increased, and it has received more and more attention. The relationship between the two has also been a hot topic in clinical research. Some foreign scholar's reported ${ }^{9}$ OSA patients prolonged esophageal clearance during nighttime sleep, leading to esophageal reflux; Ow et al $^{10}$ study confirmed that OSA severity is related to endoscopic lesions of gastro esophageal reflux, and frequent sleep apnea. There is more overlap between the stage and the longest reflux duration. The main reason is that the airway obstruction during sleep in OSA causes the negative pressure of the esophagus and thoracic cavity during inhalation, which causes the stomach contents to flow back into the esophagus and cause damage to the esophageal mucosa. A study of patients with obstructive sleep apnea syndrome found that as the obstructive sleep apnea syndrome worsens, the incidence of GERD is also elevated, namely severe obstructive sleep apnea syndrome. The incidence of GERD is higher than that of patients with moderate and mild obstructive sleep apnea syndrome.

At present, there is no simple and effective treatment for the treatment of obstructive sleep apnea syndrome. The use of CPAP in the treatment of obstructive sleep apnea syndrome is still the first-line approach, and its effectiveness has also been obtained by many physicians. Affirmative. Zhou Yingqian et al ${ }^{11}$ believe that OSA 
is a disease involving multiple mechanisms, and the single treatment is not effective. Individualized treatment strategies should be adopted for different causes, which can theoretically improve the clinical treatment effect. Lu Fangfang et al $^{12}$ It is confirmed that OSA patients have clear esophageal sphincter and esophageal sphincter pressure abnormalities, a small number of patients with GERD, and acidic substances are the main types of esophageal reflux. There is no unified treatment for patients with OSA and GERD. Kuribayashi et $\mathrm{al}^{13}$ pointed out OSA can cause nocturnal gastro- esophageal reflux at night, and continuous positive pressure ventilation can reduce nighttime GERD events in OSA patients. Cai Lianying et $\mathrm{al}^{14}$ found that GERD is associated with obstructive sleep syndrome, and obstructive sleep apnea hypopnea Patients with syndrome with GERD, nasal continuous positive airway pressure combined with anti-reflux drugs $A$ therapeutically effective. Zheng Poem light or the like. ${ }^{15}$ A study of 48 elderly OSHAS patients with GERD found that elderly patients with OSA and GERD received nasal continuous positive airway pressure combined with antireflux medication compared with nasal continuous positive airway pressure therapy or antiretroviral therapy alone.

Flow drug therapy is more effective. $R E$ is endoscopic inflammatory positive GERD, which is valued because of its potential for esophageal cancer. Does OSA increase the incidence of RE cancer? There is no clinical report, early diagnosis and early stage. Treatment can significantly improve the clinical cure rate of patients with erosive esophagitis, and effectively reduce the probability of cancer. This study found that 23 patients with OSA combined with RE found that non-invasive ventilator in combination with proton pump inhibitor in the treatment of patients with OSA combined with RE. The effect was significantly higher than that of proton pump inhibitor alone. The difference in endoscopic inflammation efficiency and $\triangle$ GERD scale between the two groups was statistically significant $(P<0.05)$. OSH combined with RE patients with noninvasive breathing when using noninvasive ventilator Opportunities increase intrathoracic pressure in patients with OSA, resulting in elevated esophageal pressure. In addition, non-invasive ventilation can reduce nighttime sensation in OSA patients. The number of times and the relaxation time of the lower esophageal sphincter reduce or prevent the gastric contents from flowing back into the esophagus, which in turn improves the reflux symptoms and esophageal mucosal lesions in patients with OSA and RE, but the sample size is small, and the mechanism needs further Research, still need to accumulate more clinical data to improve.

\section{CONCLUSION}

This study found that with the severity of OSA patients, the incidence of esophageal reflux symptoms or esophageal mucosal injury increases, combined with the treatment of proton pump inhibitors and non-invasive ventilator treatment have a certain clinical effect.

Copyright@ 15 Aug, 2019.

\section{REFERENCES}

1. Guidelines for the diagnosis and treatment of obstructive sleep apnea hypopnea syndrome (basic version) writing. Guidelines for the diagnosis and treatment of hypopnea hypopnea syndrome (basic version). Chinese Journal of General Practitioners 2015; 1 : 509-515.

2. Qi Haifeng. Changes and significance of serum irregular chemokine fractalkine in patients with moderate to severe obstructive sleep apnea hypopnea syndrome. Chinese Journal of Lung Diseases (electronic version). 2018; 1:97-99.

3. Liu Hongqin, Cai Mingjun. A case of obstructive sleep apnea hypopnea syndrome caused by hiatal hernia. Chinese Journal of Gastroesophageal Reflux Disease. 2015; 2:245-247.

4. Xu Yuying. Research progress on the relationship between chronic obstructive pulmonary disease and gastroesophageal reflux disease. Chinese Journal of Gastroesophageal Reflux Disease. 2017; 4:77-80.

5. Kaisell Ai Zhe, Aikebaier Ai Li, Ke Limu. A Budu Yi Yimu. Progress in the study of the relationship between gastroesophageal reflux disease and obesity and its complications. Chinese gastroesophageal reflux Journal of Diseases. 2016; 3:187-189. 
6. Du Guodong, Ma Lei, Lü Yunhui, Huang Lihua, Fan Chongyang, Xiang Yan, Lei Qiang, Hu Rong. Metaanalysis of the correlation between OSAHS and COPD in Chinese population. Journal of Clinical Otorhinolaryngology Head and Neck Surgery. 2016; 20:1620-1625.

7. Ji Feng, Wang Zhongyu, Han Xinyu, Li Zhitong, Wang $\mathrm{Li}$, et al. Effect of fundoplication on gastroesophageal reflux disease in obstructive sleep apnea syndrome. Chinese Journal of General Surgery. 2016; 31: 820-823.

8. Chinese Medical Association Digestive Diseases Branch. Consensus opinion of Chinese gastroesophageal reflux disease experts in 2014. Chinese Journal of Digestion. 2014; 10:649-661.

9. Suzuki M , Saigusa $H$, Kurogi $R$, Yamamoto $T$, Ishiguro T, Yohsizawa T, Kuyama Y, Furukawa T. Arousals in obstructive sleep apnea syndrome with laryngopharyngeal and gastroesophageal reflux disease. Sleep Med. 2010; 11:356-360. PubMed

10. The WC ORR, Robert JJ, Houck the JR, Giddens CL, the MM TAWK of The Effect of acid suppression ON Anatomy and Upper Airway obstruction in patients with gastroesophageal reflux and of apnea to SLEEP s disease. J Clin Sleep Med 2009; .5: 330-334. PubMed
11. Zhou Yingqian, Ye Jingying. Pathogenesis of obstructive sleep apnea hypopnea syndrome and corresponding individualized treatment strategies. Chinese Journal of Otorhinolaryngology Head and Neck Surgery. 2016; 51: 877-880.

12. Lu Fangfang, He Yongjun, Pang Yanling, Lu Lifen, Wang Lanlan, Ma Haiying. Correlation between obstructive sleep apnea syndrome and gastroesophageal reflux disease. Modern digestion and interventional therapy. 2017; 22:349-350.

13. Kuribayashi S, Kusano M, Kawamura O, Shimoyama Y, Maeda M, Hisada T, Ishizuka T, Dobashi K, Mori M. Mechanism of gastroesophageal reflux in patients with obstructive sleep apnea syndrome. Neurogastroenterol Motil. 2010; 22:611e172. PubMed

14. Cai Lianying, Zhang Facan, Liu Jianhong. Relationship between obstructive sleep apnea hypopnea syndrome and gastroesophageal reflux disease. Guangxi Medical Journal. 2005; 27:17101711.

15. Zheng Shiguang, Guo Yonghong, Tan Pan, Long Limin. Therapeutic effect of combined therapy on obstructive sleep apnea hypopnea syndrome with gastroesophageal reflux disease. Practical Geriatrics. 2013; 2: 111-113.

\begin{tabular}{|c|c|c|c|}
\hline \multicolumn{2}{|c}{ AUTHORSHIP AND CONTRIBUTION DECLARATION } \\
\hline Sr. \# & Author(s) Full Name & \multicolumn{1}{|c|}{ Contribution to the paper } & Author(s) Signature \\
\hline 1 & Umar Usman & Article writing, Data collection. \\
\hline 2 & Mohsin Riaz Askri & $\begin{array}{l}\text { Statistical analysis, References } \\
\text { writing, Literature review. } \\
\text { Result interpretation, Proof } \\
\text { reading, Technical review. }\end{array}$ \\
\hline 3 & Shumyala Maqbool & \\
\hline
\end{tabular}

